\title{
Exact Analytic Formulae of Beam Pointing Based on Achromatic Risley Prisms
}

\author{
ZhouShupeng $^{1, a^{*}, \text { Li Jinying }}{ }^{2, b}$, Wang Qiang ${ }^{3, c}$ \\ ${ }^{1}$ Institute of Optics and Electronics, Chinese Academy of Sciences, Chengdu, Sichuan, China \\ Key Laboratory of Optical Engineering, Chinese Academy of Sciences, Chengdu, Sichuan, China \\ University of Chinese Academy of Sciences, Beijing, China \\ ${ }^{2}$ Institute of Optics and Electronics, Chinese Academy of Sciences, Chengdu, Sichuan, China \\ Key Laboratory of Optical Engineering, Chinese Academy of Sciences, Chengdu, Sichuan, China \\ ${ }^{3}$ Institute of Optics and Electronics, Chinese Academy of Sciences, Chengdu, Sichuan, China \\ Key Laboratory of Optical Engineering, Chinese Academy of Sciences, Chengdu, Sichuan, China \\ azhoushupeng309@qq.com, bjyliok@163.com, ${ }^{\mathrm{c}}$ wangqiang19750731@126.com
}

\begin{abstract}
Keywords: achromatic risley prisms, beampointing, analytic formulae,vectorial law of refraction, high precision, widesteering angle

Abstract. The mathematic relationship between the given orientations of two-prism groups and the corresponding pointing position of outgoing beam is the basic prerequisite needed in applications of achromatic risley prisms to steer beam in free space. Based on vectorial law of refraction, this paper finishes the derivation of the analytic formulae of the relationship wanted. Then the test samplesobtained by the analytic formulae, the method of paraxial approximation and professional the optical software are compared respectively. The results prove the correctness of the analytic formulae, as well as its necessity and superiority in high-precision pointing solution when beam is steered over wide angular range.
\end{abstract}

\section{Introduction}

Traditionally, beampointing mechanism in photoelectric acquisition, tracking and pointing system is composed of gimbal and fast steering mirror mechanism together, in order to meet the demands of wide scan angular range, fast response frequency and high pointing precision simultaneously. Gimbal mechanism has advantage in wide scan angular range, but is dragged in the performance of dynamic movement for its big volume and inertia[1,2,3,4]. On the contrary, fast steering mirror mechanism does well in fast response frequency and high pointing precision. However, those superiorities can only be kept within really narrow scan angular range[5,6].Consist of the first and second refraction prism sharing the same spin axis, risley prisms mechanism realizesbeamspatial movement by alteringorientationsof two prisms. With relative wide scan angular range, fast responsefrequency, high pointing precision and compact simple structure, it can satisfy all those demands mentioned above in a single apparatus [7], thus showing strong comparative advantages and obvious potential for substitution [8] in many fields like photoelectric detection, space optical communication, fiber optic switchand so on. Applying risley prisms, the Geoscience Laser Altimeter System launched in 2003 achieves precise pointing of laser beam [9]. The beamsteering device developed by OPTRA Inc., which can reach pointing deviation under 1 milliradianover steering angular rangeof $60^{\circ}$, is based on risley prisms [4]. So dose the inter satellite laser communication device designed by SIOM of Chinese Academy of Sciences, which reaches scanning accuracy under 50 microradianwithinhorizontal and vertical steering angular range of $\pm 15^{\circ}[10]$.

The mathematic relationship between the given orientations of two prisms and the corresponding pointing position of outgoing beam is the prerequisite needed in applications of risley prisms to steer beam in free space.[11]. Method of paraxial approximation is adopted in solving optical refraction usually. However, it has obvious deviation of pointing solution when beam is steered in wide angel, which goes against the superiorities of risley prisms. Another method is curve fitting 
with discrete sample points. Helped by professional optical software like ZEMAX, a set of sample solutionsin very high precision can be obtained. Unfortunately, fitting doesn't work well globally under the influence of highly nonlinear characteristic existing in the relationship wanted.

Vectorial law of refraction can deal with the problem above. Subject to complex mathematic techniques, only recently the analytic formulae of the relationship wanted was derivated by Yang Y and Li Y for different risley prisms structure respectively [11,12]. Nevertheless, those formulae are extracted from structures without achromatic optical apparatus, which means they are available for scenarios using single-wave beammerely. Considering so many applying scenarios of risley prisms in need of multi-wave beam, and increase of solution complexity brought by additional achromatic optical apparatus, it is important and significant to derive exact analytic formulae that can be used in pointing solution of achromatic risley prisms.

Based on vectorial law of refraction, this paper focuses on the process of deriving the analytic formulae of the mathematic relationship wanted. And it illustrates the test samples obtained by the analytic formulae, the method of paraxial approximation and the professional optical software ZEMAX to provethe correctness of the formulae. Then the analysis of advantages of different methods are given to demonstratethe necessity and superiority of the analytic formulae in high-precision pointing solution when beam is steered over wide angular range.

\section{Structure of achromatic risley prisms}

Fig. 1 shows the achromatic risley prisms mechanism, four prisms rotate around the shared spin axis (axis-Z). Primarily, risley prisms mechanism is composed of the right-angled prisms $\Pi_{2}$ and $\Pi_{3}$ only,but now the trapezoid prisms $\Pi_{1}$ and $\Pi_{4}$ are added for compensating chromatic aberration. So theprisms $\Pi_{2}$ and $\Pi_{3}$ are called primary prisms while the prisms $\Pi_{1}$ and $\Pi_{4}$ are called achromatic prisms.

The orthogonal surfaces ofthe primary prisms $\Pi_{2}$ and $\Pi_{3}$ are parallel mutually and perpendicular to the spin axis. $\Pi_{2}$ and $\Pi_{3}$ are provided with the same open angel $\alpha_{1}$ and refraction index $n_{1}$. The additional achromatic prisms $\Pi_{1}$ and $\Pi_{4}$ are mirrored placed and provided with the same open angle $\alpha_{2}$ and refraction index $n_{2}$. Besides, their surfaces close to $\Pi_{2}$ and $\Pi_{3}$ are parallel to the inclined surfaces of the $\Pi_{2}$ and $\Pi_{3}$ respectively.

In movement, the achromatic prism $\Pi_{1}$ and the primary prism $\Pi_{2}$ shared the same rotating angle $\theta_{1}$ around the spin axis. And the achromatic prism $\Pi_{4}$ and the primary prism $\Pi_{3}$ shared rotating angle $\theta_{2}$ in a similar state. Angle $\theta_{1}$ and $\theta_{2}$ are measured from the positive axis-X by the vertex of the prism $\Pi_{2}$ and $\Pi_{3}$ respectively. Incidence beamis injected reversely along the axis- $Z$, and outgoing beamare described by azimuth angle $\Theta$ measured from the positive axis-X and elevation angle $\Phi$ measured from the negative axis-Z. 


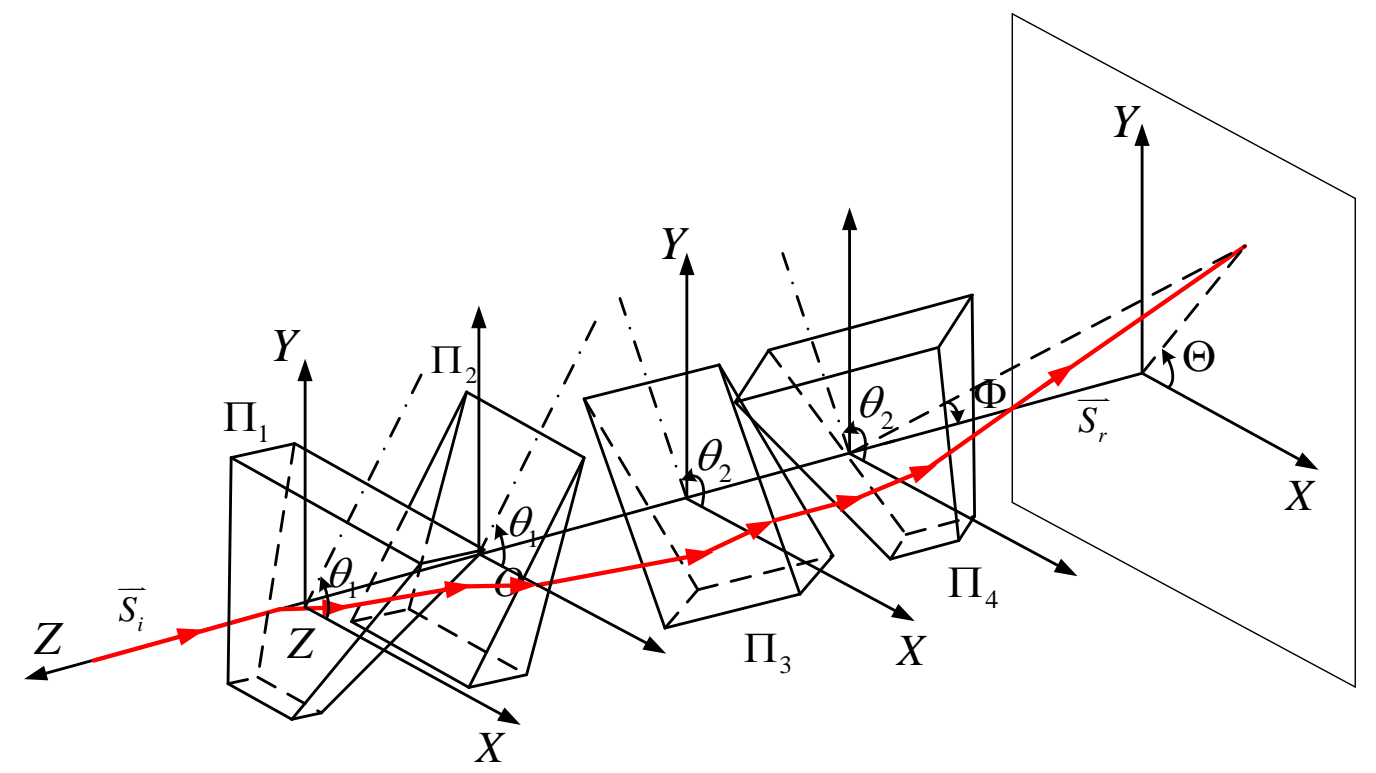

Fig.1 structure of the achromatic risley prisms mechanism

\section{Method of paraxial approximation}

Method of paraxial approximation treats prism as wedge with small open angle and supposesbeamis refracted to the thicker part of prism regardless of the position of incidence beamand the orientation of prisms. Paralleled mutuallyon the opposite surfaces,the achromatic prism $\Pi_{1}$ and theprimary prism $\Pi_{2}$ are fixed placed and rotate around the axis-Z with shared angle $\theta_{1}$. As Fig.2 shows, benefit from some known results [13], thesteered angle $\Psi_{1}$ of beampassing throughthe two prisms is represented as

$$
\Psi_{1}=\left(n_{1}-1\right)_{1} \alpha_{1}-\left(n_{2}-1\right) \alpha_{2}
$$

Similarly, thesteered angle $\Psi_{2}$ of beampassing throughthe primary prism $\Pi_{3}$ and achromatic prism $\Pi_{4}$ is given by:

$$
\Psi_{2}=\left(n_{1}-1\right)_{1} \alpha_{1}-\left(n_{2}-1\right) \alpha_{2}
$$
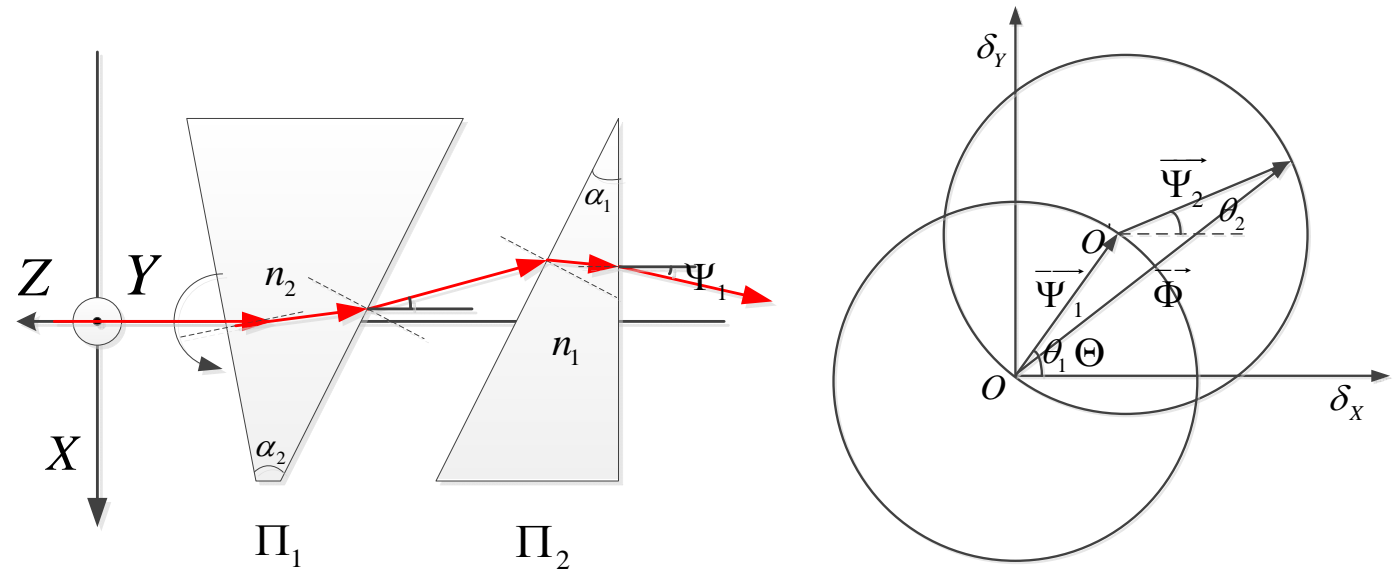

Fig.2 position of the $\Pi_{1}$ and $\Pi_{2}$ Fig.3 central algorithm

Fig. 3 shows how to obtain the pointing position of outgoing beamafter all the refractions with central algorithm [13]. Point O represents the spin axis (axis-Z) of the mechanism, and coordinate axis- $\delta_{X}, \delta_{Y}$ represents the projection of the orientation of beamon the axis-X and axis-Y respectively. Then spatial beamcan be described as a plane vector in the coordinate while the vector module represents elevation (angle between outgoing beamand the negative axis-Z) and the angle 
between the vector and axis- $\delta_{X}$ represents azimuth (orientation of outgoing beamfrom the positive axis-X) [13].

Incident from the negative axis- $Z$, the pointing position of intermediate beampassing throughthe achromatic prism $\Pi_{1}$ and the primary prism $\Pi_{2}$ is represented as a plane vector $\overrightarrow{\Psi_{1}}$. Along with rotation of the first prism group, $\overrightarrow{\Psi_{1}}$ originates from point $O$ and terminates on moving placement marked as $O^{\prime}$ of a circular trace with radius $\Psi_{1}$ of Eq.1. Then based on the intermediate beam, the plane vector $\overrightarrow{\Psi_{2}}$ describes the pointing position of ultimate beamwhich passes through the primary prism $\Pi_{3}$ and the achromatic prism $\Pi_{4}$ further. $\overrightarrow{\Psi_{2}}$ starts from $O^{\prime}$ and ends in a circular moving trace with radius $\Psi_{2}$ of Eq.2 as the second prismgroup rotating. Finally and globally, the total pointing position of outgoing beambased on the originalspin axis- $\mathrm{Z}$ is regarded as vector sum $\vec{\Phi}$ from $\overrightarrow{\Psi_{1}}$ and $\overrightarrow{\Psi_{2}}$. Projection of $\vec{\Phi}$ on the axis- $\delta_{X}$ and $\delta_{Y}$ are given by:

$$
\begin{aligned}
& \Phi_{x}=\Psi_{1} \cos \theta_{1}+\Psi_{2} \cos \theta_{2} \\
& \Phi_{y}=\Psi_{1} \sin \theta_{1}+\Psi_{2} \sin \theta_{2}
\end{aligned}
$$

From Eq.3 and Eq.4, the elevation angle $\Phi$ and the azimuth $\Theta$ are given by:

$$
\begin{aligned}
& \Phi=\sqrt{\Psi_{1}^{2}+\Psi_{2}^{2}+2 \Psi_{1} \Psi_{2} \cos \left(\theta_{1}-\theta_{2}\right)} \\
& \tan \Theta=\frac{\Psi_{1} \sin \theta_{1}+\Psi_{2} \sin \theta_{2}}{\Psi_{1} \cos \theta_{1}+\Psi_{2} \cos \theta_{2}}
\end{aligned}
$$

\section{Analytic formulae based on the vectorial law of refraction}

Unit vector $\vec{s}_{i}$ and $\vec{n}$ representincidence beamand normal vector of refraction surface respectively.The outgoing beamafter transmitting from medium of index $n_{1}$ to medium of index $n_{2}$ is described as $\vec{s}_{r}$ below. This equation is called vectorial law of refraction.

$$
\vec{s}_{r}=\frac{n_{1}}{n_{2}}\left[\vec{s}_{i}-\left(\vec{s}_{i} \cdot \vec{n}\right) \cdot \vec{n}\right]-\sqrt{1-\frac{n_{1}^{2}}{n_{2}^{2}}+\frac{n_{1}^{2}}{n_{2}^{2}}\left(\vec{s}_{i} \cdot \vec{n}\right)^{2}} \cdot \vec{n}
$$

Fig. 4 shows the refraction process of incidence beamfrom the negative axis-Z. And the normal vector of each surface is listed in order of refraction as follow:

$$
\begin{aligned}
& \vec{n}_{1}=\left(\sin \left(\alpha_{2}-\alpha_{1}\right) \cos \theta_{1}, \sin \left(\alpha_{2}-\alpha_{1}\right) \sin \theta_{1}, \cos \left(\alpha_{2}-\alpha_{1}\right)\right) \\
& \vec{n}_{2}=\left(-\sin \alpha_{1} \cos \theta_{1},-\sin \alpha_{1} \sin \theta_{1}, \cos \alpha_{1}\right) \\
& \vec{n}_{3}=\left(\sin \alpha_{1} \cos \theta_{2}, \sin \alpha_{1} \sin \theta_{2}, \cos \alpha_{1}\right) \\
& \vec{n}_{4}=\left(-\sin \left(\alpha_{2}-\alpha_{1}\right) \cos \theta_{2},-\sin \left(\alpha_{2}-\alpha_{1}\right) \sin \theta_{2}, \cos \left(\alpha_{2}-\alpha_{1}\right)\right)
\end{aligned}
$$

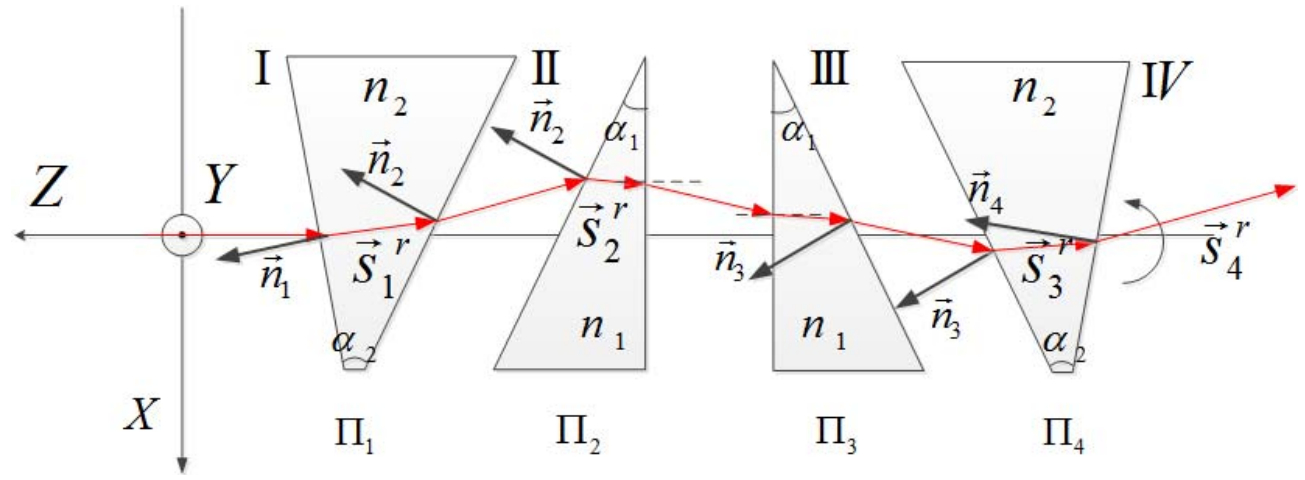

Fig.4 refractions in achromatic risley prisms mechanism 
Withvectorial law of refraction, pointing position of outgoing beamafter each refraction can be solved sequentially.

Refraction I, from the air to the achromatic prism $\Pi_{1}$. Substitute $\vec{s}_{i}=[0,0,-1]$ and Eq.8 to Eq.7, the pointing position of beamafter Refraction $\mathrm{I}$ is given by:

$$
\vec{s}_{1}^{r}=\frac{1}{n_{2}}\left[a_{1} \sin \left(\alpha_{2}-\alpha_{1}\right) \cos \theta_{1}, a_{1} \sin \left(\alpha_{2}-\alpha_{1}\right) \sin \theta_{1},-a_{2}\right]
$$

Where constants $a_{1}$ and $a_{2}$ are:

$$
\begin{aligned}
& a_{1}=\cos \left(\alpha_{2}-\alpha_{1}\right)-\sqrt{n_{2}^{2}-\sin ^{2}\left(\alpha_{2}-\alpha_{1}\right)} \\
& a_{2}=\sin ^{2}\left(\alpha_{2}-\alpha_{1}\right)+\sqrt{n_{2}^{2}-\sin ^{2}\left(\alpha_{2}-\alpha_{1}\right)} \cos \left(\alpha_{2}-\alpha_{1}\right)
\end{aligned}
$$

Refraction II , from the achromatic prism $\Pi_{1}$ to the primary prism $\Pi_{2}$. Considering parallelism of the right surface of the prism $\Pi_{1}$ and the left surface of theprism $\Pi_{2}$ would not alter transmission direction ofbeam, the two refractions taking place on those surfaces can be simplified into one from medium of index $n_{2}$ to medium of index $n_{1}$ directly. Substitute $\vec{s}_{i}=\overrightarrow{s_{1}^{r}}$ and Eq.9 to Eq.7, the pointing position of this step is given by:

$$
\vec{s}_{2}^{r}=\left\{c_{1} \cos \theta_{1}, c_{1} \sin \theta_{1}, c_{2}\right\}
$$

Where constants $b_{1}, b_{2}, b_{3}, c_{1}$ and $c_{2}$ are:

$$
\begin{aligned}
b_{1}= & \sin \left(\alpha_{2}-\alpha_{1}\right) \sin \alpha_{1}\left[\cos \left(\alpha_{2}-\alpha_{1}\right)-\sqrt{n_{2}^{2}-\sin ^{2}\left(\alpha_{2}-\alpha_{1}\right)}\right] \\
& +\cos \alpha_{1}\left[\sin ^{2}\left(\alpha_{2}-\alpha_{1}\right)+\sqrt{n_{2}^{2}-\sin ^{2}\left(\alpha_{2}-\alpha_{1}\right)} \cos \left(\alpha_{2}-\alpha_{1}\right)\right] \\
b_{2}= & b_{1}-\sqrt{1-n_{2}^{2}+b_{1}^{2}} \\
b_{3}= & \frac{\sqrt{1-n_{2}^{2}+b_{1}^{2}}}{n_{1}}-\sqrt{1-\frac{1}{n_{1}^{2}}+\frac{1}{n_{1}^{2}}\left(1-n_{2}^{2}+b_{1}^{2}\right)} \\
c_{1}= & \frac{1}{n_{1}}\left(a_{1} \sin \left(\alpha_{2}-\alpha_{1}\right)-b_{2} \sin \alpha_{1}\right)-b_{3} \sin \alpha_{1} \\
c_{2}= & \frac{b_{2}}{n_{1}} \cos \alpha_{1}-\frac{a_{2}}{n_{1}}+b_{3} \cos \alpha_{1}
\end{aligned}
$$

Refraction III, from the primary prism $\Pi_{2}$ to the achromatic prism $\Pi_{4}$. Sharing the same index $n_{1}$, the orthogonal surfaces of the prism $\Pi_{2}$ and $\Pi_{3}$ are parallel mutually and perpendicular to the spin axis (axis-Z). So their relative rotation would not alter transmission direction of beam, which means the two refractions taking place there can be neglected. Besides, similar simplification like the refraction II can be applied to the parallelism of the prisms $\Pi_{3}$ and $\Pi_{4}$. Thus the two original refractions are regarded as one from medium of index $n_{1}$ to medium of index $n_{2}$ directly. Substitute $\vec{s}_{i}=\overrightarrow{s_{2}^{r}}$ and Eq.10 to Eq.7, the pointing position of this step is represented as:

$$
\vec{s}_{3}^{r}=\left\{\frac{n_{1} c_{1}}{n_{2}} \cos \theta_{1}-\frac{g(\Delta \theta)}{n_{2}} \sin \alpha_{1} \cos \theta_{2}, \frac{n_{1} c_{1}}{n_{2}} \sin \theta_{1}-\frac{g(\Delta \theta)}{n_{2}} \sin \alpha_{1} \sin \theta_{2}, \frac{n_{1} c_{2}}{n_{2}}-\frac{g(\Delta \theta)}{n_{2}} \cos \alpha_{1}\right\}
$$

Where correlation variables $f(\Delta \theta)$ and $g(\Delta \theta)$ are:

$$
\begin{aligned}
& f(\Delta \theta)=c_{1} \sin \alpha_{1} \cos \Delta \theta+c_{2} \cos \alpha_{1}, \quad \Delta \theta=\theta_{1}-\theta_{2} \\
& g(\Delta \theta)=n_{1} f(\Delta \theta)+\sqrt{n_{2}^{2}-n_{1}^{2}+n_{1}^{2} f^{2}(\Delta \theta)}
\end{aligned}
$$

Refraction IV , from the achromatic prism $\Pi_{4}$ to the air. Substitute $\vec{s}_{i}=\overrightarrow{s_{3}^{r}}$ and Eq.11 to Eq.7, 
the pointing position of the last step is represented as:

$$
\begin{aligned}
\vec{s}_{4}^{r}=\{ & n_{1} c_{1} \cos \theta_{1}-g(\Delta \theta) \sin \alpha_{1} \cos \theta_{2}+p(\Delta \theta) \sin \left(\alpha_{2}-\alpha_{1}\right) \cos \theta_{2}, \\
& n_{1} c_{1} \sin \theta_{1}-g(\Delta \theta) \sin \alpha_{1} \sin \theta_{2}+p(\Delta \theta) \sin \left(\alpha_{2}-\alpha_{1}\right) \sin \theta_{2}, \\
& \left.n_{1} c_{2}-g(\Delta \theta) \cos \alpha_{1}-p(\Delta \theta) \cos \left(\alpha_{2}-\alpha_{1}\right)\right\}
\end{aligned}
$$

Where correlation variables $h(\Delta \theta)$ and $p(\Delta \theta)$ are:

$$
\begin{aligned}
& h(\Delta \theta)=-n_{1} c_{1} \sin \left(\alpha_{2}-\alpha_{1}\right) \cos \Delta \theta+n_{1} c_{2} \cos \left(\alpha_{2}-\alpha_{1}\right)-g(\Delta \theta) \cos \alpha_{2} \\
& p(\Delta \theta)=h(\Delta \theta)+\sqrt{1-n_{2}^{2}+h^{2}(\Delta \theta)}
\end{aligned}
$$

Above all, direction cosine $(K, L, M)$ of the pointing position of outgoing beamare:

$$
\begin{aligned}
& K=\frac{n_{1} c_{1} \cos \theta_{1}+g(\Delta \theta) \sin \alpha_{1} \cos \theta_{2}-p(\Delta \theta) \sin \left(\alpha_{2}-\alpha_{1}\right) \cos \theta_{2}}{q(\Delta \theta)-r(\Delta \theta) \cos \Delta \theta-s(\Delta \theta)} \\
& L=\frac{n_{1} c_{1} \sin \theta_{1}+g(\Delta \theta) \sin \alpha_{1} \sin \theta_{2}-p(\Delta \theta) \sin \left(\alpha_{2}-\alpha_{1}\right) \sin \theta_{2}}{q(\Delta \theta)-r(\Delta \theta) \cos \Delta \theta-s(\Delta \theta)} \\
& M=\frac{n_{1} c_{2}-g(\Delta \theta) \cos \alpha_{1}-p(\Delta \theta) \cos \left(\alpha_{2}-\alpha_{1}\right)}{q(\Delta \theta)-r(\Delta \theta) \cos \Delta \theta-s(\Delta \theta)}(16.3)
\end{aligned}
$$

Where correlation variables $q(\Delta \theta), r(\Delta \theta)$ and $s(\Delta \theta)$ are:

$$
\begin{aligned}
& q(\Delta \theta)=n_{1}^{2}\left(c_{1}^{2}+c_{2}^{2}\right)+g^{2}(\Delta \theta)+p^{2}(\Delta \theta)+2 g(\Delta \theta) p(\Delta \theta) \cos \alpha_{2} \\
& r(\Delta \theta)=2 n_{1} c_{1}\left[g(\Delta \theta) \sin \alpha_{1}-p(\Delta \theta) \sin \left(\alpha_{2}-\alpha_{1}\right)\right] \\
& s(\Delta \theta)=2 n_{1} c_{2}\left[g(\Delta \theta) \cos \alpha_{1}+p(\Delta \theta) \cos \left(\alpha_{2}-\alpha_{1}\right)\right]
\end{aligned}
$$

From Eq.16, theelevation angle $\Phi$ and the azimuth $\Theta$ of the outgoing beamare given by:

$$
\begin{aligned}
& \Phi=\arccos (-M)(17) \\
& \Theta=\left\{\begin{array}{l}
\arctan \left(\frac{L}{K}\right) ; \text { when } \mathrm{K} \geq 0 \text { and } L \geq 0 \\
\arctan \left(\frac{L}{K}\right)+2 \pi ; \text { when } \mathrm{K} \geq 0 \text { and } \mathrm{L}<0 \\
\arctan \left(\frac{L}{K}\right)+\pi ; \text { when } \mathrm{K}<0
\end{array}\right.
\end{aligned}
$$

\section{Inspection and analysis}

As a highly professional and widely applicated commercial optical software, the precision of ZEMAXin beampointing solution is guaranteed. In order to obtain more accurate beampointing solution referenceover wide steering angular range, this paper utilizes ZEMAX to simulate the process of beam refraction and the results of pointing solution. After building corresponding achromatic risley prisms model in ZEMAX, reference samples are computed by giving several couples of testing orientations $\theta_{1}$ and $\theta_{2}$ of two-prism groups. The beamused in ZEMAX simulation is single-wave because this paper focuses on verifying the correctness and superiorities of the analytic formulaeinpointing solution rather than optical design problem of achromatic process. For beamin any wavelength and medium of any index, the goal of inspection can be reached only if those parameters are accordant in simulation of ZEMAX and substitution of the analytic formulae.

Pointing deviation of two beamis represented by spatial angle between their directive vectors. In fig.5, horizontal axis represents 13 sample orientations of $\Delta \theta=\theta_{1}-\theta_{2}$ overangular range of $0 \sim 2 \pi$ in step of $\pi / 6$, and vertical axis represents deviation of beam pointing solution between different methods in corresponding orientations. The blue line illustrates the deviations between the method 
of paraxial approximation and ZEMAX, whilethe red points depict the deviations between the analytic formulae and ZEMAX.

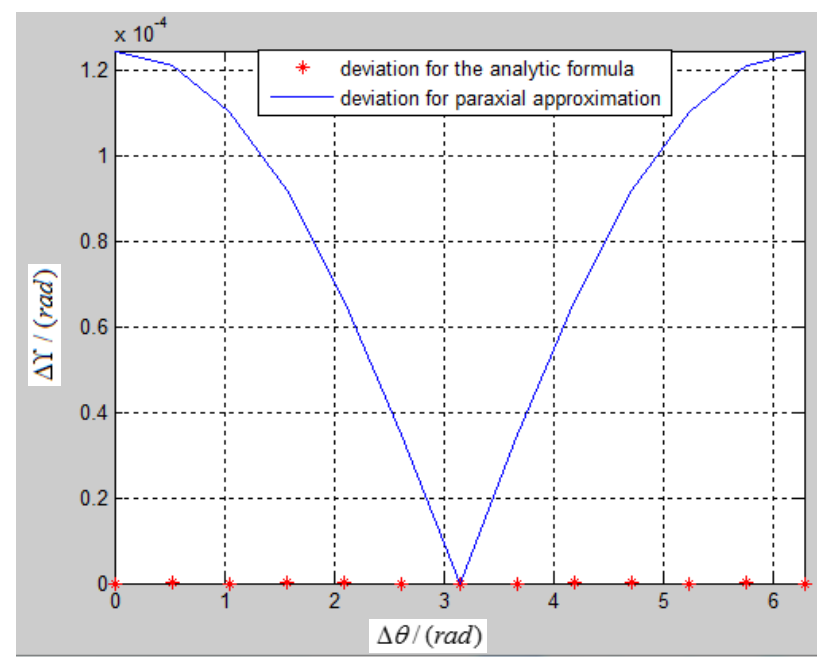

Fig.5 deviation between different methods

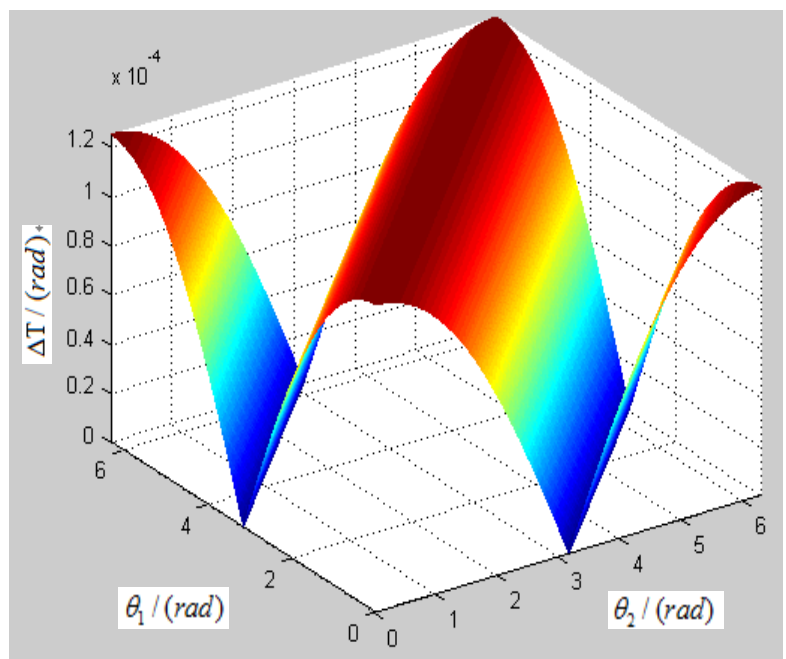

Fig.6 deviation between paraxial and analytic

For the method of paraxial approximation, only if $\Delta \theta=\pi$ can the minimum deviation 0 be reached. And the maximum deviation is on the order of $10^{-4}$ radian when $\Delta \theta$ near 0 or $2 \pi$. In general, the greater absolute value of $\Delta \theta-\pi$ is, the bigger solving deviation is.

For the analytic formulae, the minimum deviation is 0 while others are all on the order of $10^{-8}$ radian. Actually, should truncation error be eliminated, the others would be 0 as well. That is to say the result obtained by the analytic formulae is in accordance with the solution reference exactly.

Fig. 6 shows numerous deviations ofpointing solution between results obtain by paraxial approximation and the analytic formulae when $\theta_{1}$ and $\theta_{2}$ alter overangular range of $0 \sim 2 \pi$. For paraxial approximation, this figure also coincides with the change rule of solving deviation concluded before. Both the simulation of ZEMAX and Eq.5 tell that the steered angle of beam increases as the absolute value of $\Delta \theta-\pi$ grows, which means this method has obvious deviation of pointing solution when beam is steered in wide angle. So the necessity and superiority of the analytic formulae in high-precision pointing solution over wide steering angular range is proved from the reverse side.

\section{Summary}

Of the mathematic relationship between the given orientations of two-prism groups and the corresponding pointing position of outgoing beam, the analytic formulae derived from vectorial law of refraction can achieves great precision inpointing solution over wide steering angular range. Compared with the traditional method of paraxial approximation, which is simple but has obvious solving derivation when beam is steered in wide angle, the analytic formulae is more practical and reliable in scenarios demanding high precision in pointing solution while beam would be steered overwide angular range.

\section{References}

[1] SUN H, LANG X L, LI ZH Q, et al.. Measurement for LOS stabilization precision of electro-optical imaging system on moving carrier [J]. Optics and Precision Engineering, 2011, 19(9):2131-2137. (in Chinese)

[2]LI Y, CAO L H, WANG D N. Controlling of shipborne optoelectronic searching and tracking system based on inertial navigation platform [J]. Optics and Precision Engineering, 2011, 19(5):1126-1123. (in Chinese) 
[3] CHEN C B. Beam steering and pointing with counter-rotating prisms [J]. SPIE, 2007, 6714:671409.

[4] SCHWARZE C R, VAILLANCOURT R, CARLSON D, et al.. Risley prism based compact laser beam steering for IRCM, laser communications, and laser radar [EB/OL].[2012-07-11]. http://www.optra.com/images/TP-Compact-Beam-Steering.pdf.

[5] ULANDER K. Two-axis beam steering mirror control system for precision pointing and tracking applications [D]. Sanluis Obispo, CA: Faculty of California Polytechnic State University, 2006.

[6] KIM B S, GIBSON S, TSAO T. Adaptive control of a tilt mirror for laser beam steering [C]. Boston: AACC, 2004.

[7] LI A, JIANG X, SUN J, et al.. Laser coarse fine coupling scanning method by steering double prisms [J]. Appl. Opt., 2012, 51(3):356-364.

[8] GARCIA-TORALES G, FLORES J L, MUNOZ R X. High precision prism scanning system [C]. Sixth Symposium Optics in Industry. International Society for Optics and Photonics, 2007, 6442:64220X-6442X-8.

[9] AFZAL R S, YU A, DALLAS J L. The Geoscience laser Altimeter system(GLAS) laser transmitter [J]. IEEE J, Selected Topics In Quantum Electronics, 2007, 13(3):511-536.

[10] LI A, LIU L, SUN J, et al.. Research on a scanner for tilting orthogonal double prisms [J]. Appl. Opt., 2006, 45:8063-8069.

[11] YANG Y. Analytic solution of free space optical beam steering using risley prisms [J]. Chinese Optics, 2013, 6(2): 136-150. (in Chinese)

[12] LI Y. Closed form analytic inverse solutions for Risley-prism-based beam steering system [J]. Appl. Opt., 2011, 50(5): 679-686

[13] ZHOU Y, LU Y F, HEI M, et al.. Analytic solution of optical beam steering based on rotational double prisms [J]. Opt. Precision Eng., 2013, 21(6):1373-1379. (in Chinese) 